\title{
P03-136
}

\section{GAMMA BAND RESPONSE DURING AUDITORY INFORMATION PROCESSING IN SCHIZOPHRENIA}

N. Domján ${ }^{1}$, G. Csifcsák ${ }^{1,2}$, E.A. Garab ${ }^{3}$, I. Szendi ${ }^{1}$, Z. Janka

${ }^{1}$ Department of Psychiatry, ${ }^{2}$ Department of Psychology, University of Szeged, Szeged, ${ }^{3}$ Department of Cognitive Science, University of Technology and Economics, Budapest, Hungary

Aims: In the present study we investigated the correlation between evoked gamma activity and P50 suppression and between induced gamma activity and P300 amplitude on one schizophrenic population. These kind of auditory processing impairments are substantial in schizophrenia.

Methods: Thirty-one patients with schizophrenia and twenty-one controls were involved. Electroencephalogram was recorded with $19 \mathrm{Zn}$ electrodes, which were placed according to the international 10-20 system.

Results: P50 suppression was significantly reduced in schizophrenic patients $(F(1,53)=9.89, p=0.03)$. There was a not significant reduction in the early evoked gamma response. Furthermore, we found no correlation between P50 amplitudes and gamma amplitude for the first stimulus in neither groups, while there was a significant correlation for the second click in the control group $(R=0.43, p=0.03$ for controls, $R=0.18, p=0.30$ for patients). The amplitude of the $P 300$ auditory evoked potential was significantly reduced at $P z$ electrode in schizophrenic patients $(F(1,53)=11.91, p=0.01)$. Induced gamma response around the peak $P 300$ latency was diminished in the patient group $(F(1,53)=11.12, p=0.002)$. There was no correlation between $P 300$ amplitudes and gamma amplitude in neither group.

Conclusion: According to our results evoked and induced gamma activity is differentially affected in schizophrenia during auditory information processing. The absence of late induced gamma response during target stimulus detection among patients might reflect abnormal functional connectivity between different cortical areas, and result in inefficient integration and utilization of sensory stimuli. 